\title{
On Homoclinic Solutions for First-Order Superquadratic Hamiltonian Systems with Spectrum Point Zero
}

\author{
Feng $L^{1}{ }^{1}$ and Juntao Sun ${ }^{2}$ \\ ${ }^{1}$ School of Science, Linyi University, Linyi, Shandong 276005, China \\ ${ }^{2}$ School of Science, Shandong University of Technology, Zibo, Shandong 255049, China \\ Correspondence should be addressed to Feng Li; lf0539@126.com
}

Received 5 December 2013; Accepted 25 March 2014; Published 24 April 2014

Academic Editor: Yongli Song

Copyright (C) $2014 \mathrm{~F}$. Li and J. Sun. This is an open access article distributed under the Creative Commons Attribution License, which permits unrestricted use, distribution, and reproduction in any medium, provided the original work is properly cited.

The existence and multiplicity of homoclinic solutions for a class of first-order periodic Hamiltonian systems with spectrum point zero are obtained. The proof is based on two critical point theorems for strongly indefinite functionals. Some recent results are improved and extended.

\section{Introduction}

Consider the following first-order Hamiltonian systems:

$$
\dot{u}(t)=\mathscr{J} H_{u}(t, u), \quad t \in \mathbb{R},
$$

where $u=(y, z) \in \mathbb{R}^{2 N}, \mathscr{J}$ is the standard symplectic matrix in $\mathbb{R}^{2 N}$

$$
\mathscr{J}=\left(\begin{array}{cc}
0 & -I_{N} \\
I_{N} & 0
\end{array}\right),
$$

and $H \in C^{1}\left(\mathbb{R} \times \mathbb{R}^{2 N}, \mathbb{R}\right)$ has the form

$$
H(t, u)=\frac{1}{2} L u \cdot u+W(t, u)
$$

with $L \in C\left(\mathbb{R}, \mathbb{R}^{4 N^{2}}\right)$ being a $2 N \times 2 N$ symmetric matrixvalued function and $W \in C^{1}\left(\mathbb{R} \times \mathbb{R}^{2 N}, \mathbb{R}\right)$. A solution $u$ of (HS) is a homoclinic orbit if $u \neq \equiv$ and $u(t) \rightarrow 0$ as $|t| \rightarrow$ $\infty$.

To continue the discussion, we need the following notation:

$$
A:=-\left(g \frac{d}{d t}+L\right) .
$$

Homoclinic orbits of dynamical systems are important in applications for a number of reasons. They may be "organizing centers" for the dynamics in their neighborhood. From their existence one may, under certain conditions, infer the existence of chaos nearby or the bifurcation behavior of periodic orbits. As a special case of dynamical systems, Hamiltonian systems are very important in the study of gas dynamics, fluid mechanics, relativistic mechanics, and nuclear physics.

During the last decades, many authors are devoted to the study of homoclinic orbits for Hamiltonian systems via modern variational methods. For example, see [1-4] for the second-order systems and [5-16] for the first-order systems. To be precise, in 1999, Ding and Willem [15] studied the existence of homoclinic solutions for a class of the first-order periodic Hamiltonian systems (HS) with spectrum point zero under the well-known (AR) condition as follows:

$$
\exists \beta>2, \quad 0<\beta W(t, u) \leq W_{u}(t, u) u, \quad u \neq 0 .
$$

Later, under condition (4), Ding and Girardi [11] obtained that (HS) has infinitely many homoclinic orbits provided that $W(t, u)$ is even in $u$. However, there are many potentials satisfying superquadratic condition, not satisfying the (AR) condition (4).

Motivated by the above facts, in this paper, our aim is to study homoclinic solutions for (HS) under the conditions that zero is a continuous spectrum point and $W(t, u)$ satisfies weak superquadratic conditions. 
Set

$$
\begin{gathered}
\theta=(0,0, \ldots, 0) \in \mathbb{R}^{2 N} \\
\widetilde{W}(t, u)=\frac{1}{2} W_{u}(t, u) u-W(t, u) .
\end{gathered}
$$

Given a $2 N \times 2 N$ matrix $w$, we say that $w \geq 0$ if and only if

$$
\inf _{s \in \mathbb{R}^{2 N},|s|=1} w s \cdot s \geq 0
$$

Also letting $I_{2 N}$ be the identity matrix in $\mathbb{R}^{2 N}$ and $q \in \mathbb{R}$, we denote the matrix $q I_{2 N}$ by $q$. Moreover, denote by $\sigma(A)$ and $\sigma_{\text {cont }}(A)$ the spectrum and the continuous spectrum of the operator $A$, respectively.

We make the following assumptions:

$\left(\mathrm{L}_{1}\right) L(t)$ is 1-periodic on $t .0 \in \sigma(A)$ and there exists $\beta>0$ such that $(0, \beta] \cap \sigma(A)=\emptyset$.

$\left(\mathrm{H}_{1}\right) W \in C^{1}\left(\mathbb{R} \times \mathbb{R}^{2 N},[0, \infty)\right)$ is 1 -periodic in $t, W(t, 0) \equiv$ 0 .

$\left(\mathrm{H}_{2}\right) W_{u}(t, u)=o(u)$ uniformly in $t$ as $|u| \rightarrow 0$.

$\left(\mathrm{H}_{3}\right) W(t, u) /|u|^{2} \rightarrow \infty$ as $|u| \rightarrow \infty$ uniformly in $t$.

$\left(\mathrm{H}_{4}\right) \widetilde{W}(t, u):=(1 / 2) W_{u}(t, u) u-W(t, u)>0$ if $u \neq 0$, and there exist $r>0$ and $\eta>1$ such that $\left|W_{u}(t, u)\right|^{\eta} \leq$ $c_{1} \widetilde{W}(t, u)|u|^{\eta}$ if $|u| \geq r$.

Remark 1. The following functions satisfy weak superquadratic conditions $\left(\mathrm{H}_{1}\right)-\left(\mathrm{H}_{4}\right)$ but do not verify (4):

$$
\begin{aligned}
\operatorname{Ex} 1 . W(t, u)=d(t)( & \left(|u|^{p}-1\right) \\
& \left.+(p-2)|u|^{p-\epsilon} \sin ^{2}\left(\frac{|u|^{\epsilon}}{\epsilon}\right)\right),
\end{aligned}
$$

$$
\begin{aligned}
\operatorname{Ex} 2 . W(t, u)=d(t) & \left(\left(|u|^{2}-1\right) \ln (1+|u|)\right. \\
& \left.+\frac{|u|}{2}(2-|u|)\right),
\end{aligned}
$$

where $d(t)>0$ and is 1-periodic in $t$ and $p>2,0<\epsilon<p-2$.

Now we only check (Ex1.). It is easy to verify that $\left(\mathrm{H}_{1}\right)-\left(\mathrm{H}_{4}\right)$ are satisfied. However, following the discussion of Remark 1.2 in [17], let $u_{n}=(\epsilon(n \pi+3 \pi / 4))^{1 / \epsilon} I$, where $I=(1,0, \ldots, 0)$. Then for any $\beta>2$, one has

$$
\begin{aligned}
& W_{u}\left(t, u_{n}\right) u_{n}-\beta W\left(t, u_{n}\right) \\
& =d(t)\left[(p-\beta)\left|u_{n}\right|^{p}+(p-2)(p-\epsilon-\beta)\right. \\
& \times\left|u_{n}\right|^{p-\epsilon} \sin ^{2}\left(\frac{|u|^{\epsilon}}{\epsilon}\right) \\
& \left.+(p-2)\left|u_{n}\right|^{p} \sin 2\left(\frac{|u|^{\epsilon}}{\epsilon}\right)\right] \\
& =d(t)\left|u_{n}\right|^{p}[2-p \\
& \left.+\frac{(p-2)(p-\epsilon-\beta) \sin ^{2} \sin ^{2}\left(|u|^{\epsilon} / \epsilon\right)}{\left|u_{n}\right|^{\epsilon}}\right] \\
& \longrightarrow-\infty \quad \text { as } n \longrightarrow \infty .
\end{aligned}
$$

That is, condition (4) is not satisfied for $\beta>2$.

Observe that, due to the periodicity of $L$ and $W$, if $u$ is a homoclinic orbit of (HS), then so is $a * u$ for each $a \in \mathbb{Z}$, where $(a * u)(t)=u(t+a)$. Two solutions $u_{1}$ and $u_{2}$ are said to be geometrically distinct if $a * u_{1} \neq u_{2}$ for all $a \in \mathbb{Z}$.

Now we state our main result.

Theorem 2. Assume that $\left(L_{1}\right)$ and $\left(H_{1}\right)-\left(H_{4}\right)$ hold. Then $(H S)$ has at least one homoclinic orbit. In addition, if $W(t, u)$ is even in $u$, then (HS) has infinitely many geometrically distinct homoclinic orbits.

This paper is organized as follows. In Section 2, we formulate the variational setting and recall some critical point theorems required. In Section 3, we discuss linking structure and the Cerami condition of the functional. In Section 4, we prove Theorem 2.

Notation 1. Throughout the paper we will denote by $c>0$ various positive constants which may vary from line to line and are not essential to the problem.

\section{Preliminaries}

In what follows by $|\cdot|_{q}$ we denote the usual $L^{q}$-norm and by $(\cdot, \cdot)_{2}$ the usual inner product of $L^{2}\left(\mathbb{R}, \mathbb{R}^{2 N}\right)$. A standard Floquet reduction argument shows that $\sigma(A)=\sigma_{\text {cont }}(A)$ (see Proposition 2.2 in [15]).

Let $\{F(\lambda) ; \lambda \in \mathbb{R}\}$ be the spectral family of $A$. We have $A=U|A|$, called the polar decomposition, where $U=I-$ $F(0)-F(-0)$. By $\left(\mathrm{L}_{1}\right), L^{2}$ has an orthogonal decomposition

$$
L^{2}=L^{2-} \oplus L^{2+},
$$

where $L^{2 \pm}:=\left\{u \in L^{2} ; U u= \pm u\right\}$. 
Let $E$ be the linear space of the completion of $\mathscr{D}\left(|A|^{1 / 2}\right)$ under the norm

$$
\|u\|_{0}:=\left.\left.|| A\right|^{1 / 2} u\right|_{2} .
$$

Then $E$ is a Hilbert space under the inner product

$$
(u, v)_{0}:=\left(|A|^{1 / 2} u,|A|^{1 / 2} v\right)_{2} .
$$

E possesses an orthogonal decomposition

$$
E=E^{-} \oplus E^{+},
$$

where $E^{ \pm} \supseteq L^{2 \pm} \cap \mathscr{D}\left(|A|^{1 / 2}\right)$, the corresponding projections being denoted by $P^{+}, P^{-}$.

By $\left(\mathrm{L}_{1}\right)$, it is easy to check

$$
E^{+}=L^{2+} \cap \mathscr{D}\left(|A|^{1 / 2}\right), \quad\|\cdot\|_{0} \sim\|\cdot\|_{H^{1 / 2}} \quad \text { on } E^{+},
$$

where " " means "equivalence." Therefore, $E^{+}$can be embedded continuously into $L^{\mu}\left(\mathbb{R}, \mathbb{R}^{2 N}\right)$ for any $\mu \geq 2$ and compactly into $L_{\text {loc }}^{\mu}\left(\mathbb{R}, \mathbb{R}^{2 N}\right)$ for any $\mu \in[2, \infty)$.

However, since 0 may belong to a spectrum of $A$, then $\|\cdot\|_{0}$ may not be equivalent to $H^{1 / 2}$-norm on $E^{-}$. Therefore, in the following we use the spectrum family of $A$ to separate $\sigma(A) \cap(\infty, 0]$ into two segments. That is, for any $\epsilon>0$, set

$$
L_{\epsilon}^{2-}=F(-\epsilon) L^{2}
$$

and $E_{\epsilon}^{-}=L_{\epsilon}^{2-} \cap \mathscr{D}\left(|A|^{1 / 2}\right)=L_{\epsilon}^{2-} \cap E^{-}$. Let $\widetilde{L}_{\epsilon}^{2-}:=L^{2-} \ominus L_{\epsilon}^{2-}=$ $L^{2-} \cap\left(c l_{L^{2}}\left(\cup_{\lambda<-\epsilon} F(\lambda) L^{2}\right)\right)^{\perp}$, where $c l_{L^{2}}(M)$ denotes the closure of the set $M$ in $L^{2}$. Similar to $E^{+}$, since the spectrum of $A$ restricted to $E_{\epsilon}^{-}$is bounded away from 0 , thus, one has

$$
\|\cdot\|_{0} \sim\|\cdot\|_{H^{1 / 2}} \text { on } E_{\epsilon}^{-} .
$$

However, $\widetilde{L}_{\epsilon}^{2-}$ is not complete with respect to the norm $\|\cdot\|_{0}$; thus it is reasonable to introduce a new norm. Define

$$
\|u\|=\left(\left.\left.|| A\right|^{1 / 2} u\right|_{2} ^{2}+|u|_{2}^{2}\right)^{1 / 2}
$$

and let $E_{\epsilon, 2}^{-}$be the completion of $\widetilde{L}_{\epsilon}^{2-}$ under the norm $\|\cdot\|$.

Lemma 3 (see [16], Lemma 2.1). $E_{\epsilon, 2}^{-} \subset H_{\mathrm{loc}}^{1}$ and is embedded compactly in $L_{\mathrm{loc}}^{\infty}$ and continuously in $L^{\mu}$ for all $\mu \geq 2$.

Let $Q^{-}$denote the completion of $\mathscr{D}(A) \cap L^{2-}$ with respect to the norm $\|\cdot\|$. Since $H^{1 / 2}$ is continuously embedded in $L^{\mu}$ for all $\mu \geq 2$, by (15), $E_{\epsilon}^{-}$is a closed subspace of $Q^{-}$. Note that $E_{\epsilon, 2}^{-} \subset E^{-}$and is orthogonal to $E_{\epsilon}^{-}$with respect to $(\cdot, \cdot)_{0}$. Then $Q^{-}$has the following decomposition:

$$
Q^{-}=E_{\epsilon}^{-} \oplus E_{\epsilon, 2}^{-} .
$$

Let $Q$ be the completion of $\mathscr{D}(A)$ under the norm $\|\cdot\|$. We have the following lemma.
Lemma 4. Suppose that $\left(L_{1}\right)$ is satisfied. Then $Q$ has the direct sum decomposition

$$
Q=Q^{-} \oplus E^{+},
$$

and $Q$ is embedded continuously in $L^{\mu}$ and compactly in $L_{\text {loc }}^{\mu}$ for any $\mu \in[2, \infty)$.

Proof. By (13), (15), and (17) and Lemma 3, $Q^{-}$and $E^{+}$are closed, and using the decomposition of $E$, it is easy to check that $Q^{-} \cap E^{+}=\{0\}$, and so (18) holds. Using the same facts above and Lemma 3, one can obtain easily the desired conclusion on embedding.

It is easy to verify that $Q$ is a Hilbert space under the inner product $(u, v)=(u, v)_{0}+(u, v)_{2}$, and $\|\cdot\|$ is its induced norm. From now on, we consider the space $Q$ as our working space. Clearly, $\mathscr{D}\left(|A|^{1 / 2}\right) \subset Q \subset E$ and all norms $\|\cdot\|_{0},\|\cdot\|_{H^{1 / 2}}$, and $\|\cdot\|$ are equivalent to $E^{+}$. It is not difficult to check that $\|\cdot\|$ is uniformly convex, so $Q$ is reflexive. Set

$$
\varphi(u)=\int_{\mathbb{R}} W(t, u) .
$$

From Lemma 4 and (25) in Section 3, it follows that $\varphi$ is defined on the Banach space $Q$ and belongs to $C^{1}(Q, \mathbb{R})$, and

$$
\varphi^{\prime}(u) \cdot v=\int_{\mathbb{R}} W_{u}(t, u) v \quad \forall u, v \in Q .
$$

Consider the functional

$$
\Phi(u)=\frac{1}{2}\left(\left\|u^{+}\right\|_{0}^{2}-\left\|u^{-}\right\|_{0}^{2}\right)-\varphi(u)
$$

for $u=u^{-}+u^{+} \in Q$. Then $\Phi \in C^{1}(Q, \mathbb{R})$ and a standard argument shows that critical points of $\Phi$ are homoclinic orbits of (HS) (see [15]).

In order to study the critical points of $\Phi$, we now recall some abstract critical point theory developed recently in [18].

Let $E$ be a Banach space with direct sum decomposition $E=X \oplus Y$ and the corresponding projections $P_{X}, P_{Y}$ onto $X, Y$, respectively. For a functional $\Phi \in C^{1}(E ; \mathbb{R})$ we write $\Phi_{a}=\{z \in E: \Phi(z) \geq a\}, \Phi^{b}=\{z \in E: \Phi(z) \leq$ $b$, and $\Phi_{a}^{b}=\Phi_{a} \cap \Phi^{b}$. Recall that $\Phi$ is said to be weakly sequentially lower semicontinuous if for any $z_{n} \rightarrow z$ in $E$ one has $\Phi(z) \leq \lim _{\inf } f_{n \rightarrow \infty} \Phi\left(z_{n}\right) . \Phi^{\prime}$ is said to be weakly sequentially continuous if $\lim _{n \rightarrow \infty} \Phi^{\prime}\left(z_{n}\right) u=\Phi^{\prime}(z) u$ for each $u \in E$. A sequence $\left\{z_{n}\right\} \subset E$ is said to be a $(C)_{c^{-}}$ sequence if $\Phi\left(z_{n}\right) \rightarrow c$ and $\left(1+\left\|z_{n}\right\|\right) \Phi^{\prime}\left(z_{n}\right) \rightarrow 0$. $\Phi$ is said to satisfy the $(C)_{c}$-condition if any $(C)_{c}$-sequence has a convergent subsequence. A set $\mathscr{A} \subset E$ is said to be a $(C)_{c^{-}}$ attractor if for any $\varepsilon, \delta>0$ and any $(C)_{c}$-sequence $\left\{z_{j}\right\}$ there is $j_{0}$ such that $z_{j} \in U_{\varepsilon}\left(\mathscr{A} \cap \Phi_{c-\delta}^{c+\delta}\right)$ for $j \geq j_{0}$. Given an interval $I \subset \mathbb{R}, A$ is said to be a $(C)_{I}$-attractor if it is a $(C)_{c}$-attractor for all $c \in I$.

From now on, we assume that $X$ is separable and reflexive and fix a countable dense subset $\delta \subset X^{*}$. For each $s \in \mathcal{S}$ there exists a seminorm on $E$ defined by

$$
\begin{gathered}
p_{s}: E \longrightarrow \mathbb{R}, \\
p_{s}(z)=|s(x)|+\|y\| \quad \text { for } z=x+y \in X \oplus Y .
\end{gathered}
$$


We denote by $\mathscr{T}_{\mathcal{S}}$ the induced topology. Let $u^{*}$ denote the weak ${ }^{*}$-topology on $E^{*}$.

Suppose the following.

$\left(\mathcal{N}_{0}\right)$ For any $c \in \mathbb{R}, \Phi_{c}$ is $\mathscr{T}_{\delta}$-closed, and $\Phi^{\prime}:\left(\Phi_{c}, \mathscr{T}_{\delta}\right) \rightarrow$ $\left(E^{*}, u^{*}\right)$ is continuous.

$\left(\mathcal{N}_{1}\right)$ For any $c>0$, there exists $\zeta>0$ such that $\|z\|<$ $\zeta\left\|P_{Y} z\right\|$ for all $z \in \Phi_{c}$.

$\left(\mathcal{N}_{2}\right)$ There exists $\rho>0$ with $\kappa:=\inf \Phi\left(S_{\rho} Y\right)>0$, where $S_{\rho} Y:=\{z \in Y:\|z\|=\rho\}$.

$\left(\mathcal{N}_{3}\right)$ There exists an increasing sequence $Y_{n} \subset Y$ of finitedimensional subsequences and a sequence $\left\{R_{n}\right\}$ of positive numbers such that, letting $E_{n}=X \oplus Y_{n}$ and $B_{n}=B_{R_{n}} \cap E_{n}, \sup \Phi\left(E_{n}\right)<\infty$ and $\sup \Phi\left(E_{n} \backslash B_{n}\right)<$ $\inf \Phi\left(B_{\rho}^{n} \cap Y\right)$.

$\left(\mathcal{N}_{4}\right)$ For any interval $I \subset(0, \infty)$, there exists a $(C)_{I^{-}}$ attractor $A$ with $P_{X} A$ bounded and inf $\left\{\left\|P_{Y}(z-w)\right\|\right.$ : $\left.z, w \in A, P_{Y}(z-w) \neq 0\right\}>0$.

Now we state two critical point theorems which will be used later.

Theorem 5. Let $\left(\mathcal{N}_{0}\right)-\left(\mathcal{N}_{2}\right)$ be satisfied and suppose that there are $R>\rho>0$ and $e \in Y$ with $\|e\|=1$ such that $\sup \Phi(\partial Q) \leq \kappa$, where $Q=\{z=x+t e: t \geq 0, x \in X,\|z\|<R\}$. Then $\Phi$ has a $(C)_{c}$-sequence with $\kappa \leq c \leq \bar{c}:=\sup \Phi(Q)$.

Theorem 6. Assume $\Phi$ is even with $\Phi(0)=0$ and let $\left(\mathcal{N}_{0}\right)-\left(\mathcal{N}_{4}\right)$ be satisfied. Then $\Phi$ possesses an unbounded sequence of positive critical values.

\section{Linking Structure and the $(C)_{\mathcal{C}}$-Sequence}

We now study the linking structure and the $(C)_{c}$-sequence of $\Phi$. Observe that if $\left(\mathrm{H}_{4}\right)$ holds, then $\left|W_{u}(t, u)\right|^{\eta} \leq$ $c_{1}\left|W_{u}(t, u)\right||u|^{\eta+1} ;$ hence

$$
\left|W_{u}(t, u)\right| \leq c_{2}|u|^{p-1}, \quad \text { if }|u| \geq r
$$

for $p \geq \tau:=2 \eta /(\eta-1)>2$. Remark that $(23)$ and $\left(\mathrm{H}_{1}\right)-\left(\mathrm{H}_{2}\right)$ imply that, given $p \geq \tau$, for any $\varepsilon>0$, there is $C_{\varepsilon}>0$ such that

$$
\begin{gathered}
\left|W_{u}(t, u)\right| \leq \varepsilon|u|+C_{\varepsilon}|u|^{p-1} \\
|W(t, u)| \leq \varepsilon|u|^{2}+C_{\varepsilon}|u|^{p}
\end{gathered}
$$

for all $(t, u)$.

Lemma 7. Assume that $\left(H_{1}\right)-\left(H_{2}\right)$ and $\left(H_{4}\right)$ hold. Then there exists $\rho>0$ such that $\kappa:=\inf \Phi\left(S_{\rho}^{+}\right)>0$, where $S_{\rho}^{+}=\partial B_{\rho} \cap E^{+}$.

Proof. Choose $p \geq \tau$ such that (25) holds for any $\varepsilon>0$. This yields

$$
\varphi(u) \leq \varepsilon|u|_{2}^{2}+C_{\varepsilon}|u|_{p}^{p} \leq c\left(\varepsilon\|u\|^{2}+C_{\varepsilon}\|u\|^{p}\right)
$$

for all $u \in Q$; together with the equivalence of $\|\cdot\|_{0}$ and $\|\cdot\|$ on $E^{+}$, the lemma follows from the form of $\Phi$.
Set $\underline{\lambda}=\inf [\sigma(A) \cap(0, \infty)] .\left(\mathrm{L}_{1}\right)$ implies that $\underline{\lambda} \geq \beta>0$. Choose $\omega=2 \underline{\lambda}$. Then we can take a number $\bar{\lambda}$ such that

$$
\underline{\lambda}<\bar{\lambda}<\omega .
$$

Since $\sigma(A)=\sigma_{\text {cont }}(A)$, the subspace $Y_{0}:=\left(F_{\bar{\lambda}}-F_{0}\right) L^{2}$ is infinite dimensional, where $\left\{F_{\lambda}: \lambda \in \mathbb{R}\right\}$ denotes the spectral family of $A$. Note that $Y_{0} \subset E^{+}$and

$$
\underline{\lambda}|u|_{2}^{2} \leq\|u\| \leq \bar{\lambda}|u|_{2}^{2} \quad \text { for any } u \in Y_{0}
$$

Let $\left\{\alpha_{n}\right\}$ be a sequence with

$$
\underline{\lambda}=\alpha_{0}<\alpha_{1}<\alpha_{2}<\cdots \leq \bar{\lambda} .
$$

For each $n \in \mathbb{N}$, take an element $e_{n} \in\left(F_{\alpha_{n}}-F_{\alpha_{n-1}}\right) L^{2}$ with $\left\|e_{n}\right\|=1$ and define $Y_{n}:=\operatorname{span}\left\{e_{1}, \ldots, e_{n}\right\}$. Then $Y_{n} \subset Y_{0}$ is an increasing sequence of finite dimensional subsequence of $E^{+}$. Set $Q_{n}=Q^{-} \oplus Y_{n}$.

Lemma 8. Suppose that $\left(H_{1}\right)$ and $\left(H_{3}\right)-\left(H_{4}\right)$ are satisfied and $\rho>0$ is given by Lemma 7. Then sup $\Phi\left(Q_{n}\right)<\infty$, and there exists a sequence $\left\{R_{n}>0\right\}_{n \in N}$ such that $\sup \Phi\left(Q_{n} \backslash B_{n}\right)<$ $\inf \Phi\left(B_{\rho} \cap E^{+}\right)$, where $B_{n}:=\left\{z \in Q_{n}:\|z\| \leq R_{n}\right\}$.

Proof. It suffices to show that $\Phi(u) \rightarrow-\infty$ as $\|u\| \rightarrow \infty$ in $Q_{n}$. If not, we assume that, for some sequence $\left\{u_{j}\right\} \subset Q_{n}$ with $\left\|u_{j}\right\| \rightarrow \infty$, there exists $M_{0}>0$ such that $\Phi\left(u_{j}\right) \geq-M_{0}$ for all $j$. Define $v_{j}=u_{j} /\left\|u_{j}\right\|$; we have $\left\|v_{j}\right\|=1$. Passing to subsequence $v_{j} \rightarrow v, v_{j}^{-} \rightarrow v^{-}, v_{j} \rightarrow v$ in $L_{\text {loc }}^{\mu}$ for $\mu \in[2, \infty)$ and $v_{j}^{+} \rightarrow v^{+}$with respect to $\|\cdot\|$ and $\|\cdot\|_{0}$. Then, by $\left(\mathrm{H}_{1}\right)$ we have

$$
\begin{gathered}
\frac{1}{2}\left(\left\|v_{j}^{+}\right\|_{0}^{2}-\left\|v_{j}^{-}\right\|_{0}^{2}\right) \geq \frac{1}{2}\left(\left\|v_{j}^{+}\right\|_{0}^{2}-\left\|v_{j}^{-}\right\|_{0}^{2}\right) \\
-\int_{\mathbb{R}} \frac{W\left(t, u_{j}\right)}{\left\|u_{j}\right\|^{2}}=\frac{\Phi\left(u_{j}\right)}{\left\|u_{j}\right\|^{2}} \geq \frac{-M_{0}}{\left\|u_{j}\right\|^{2}} .
\end{gathered}
$$

We claim that $v^{+} \neq 0$. Indeed, if not, it follows from (30) that $\left\|v_{j}^{-}\right\|_{0} \rightarrow 0$. Also $\int_{\mathbb{R}}\left(W\left(t, u_{j}\right) /\left\|u_{j}\right\|^{2}\right) \rightarrow 0$.

By $\left(\mathrm{H}_{3}\right)$, there exists $r^{\prime}>0$ such that

$$
W(t, u) \geq \omega|u|^{2} \quad \text { if }|u|>r^{\prime} .
$$

For $0 \leq l<m$, let

$$
\Omega_{j}(l, m)=\left\{t \in \mathbb{R}: l \leq\left|u_{j}(t)\right|<m\right\} .
$$

Thus, by (31) and (32), one has

$$
\int_{\mathbb{R}} \frac{W\left(t, u_{j}\right)}{\left\|u_{j}\right\|^{2}} \geq \int_{\Omega_{j}\left(r^{\prime}, \infty\right)} \frac{W\left(t, u_{j}\right)}{\left\|u_{j}\right\|^{2}} \geq \omega \int_{\Omega_{j}\left(r^{\prime}, \infty\right)}\left|v_{j}\right|^{2} .
$$

From (33) and $\int_{\mathbb{R}}\left(W\left(t, u_{j}\right) /\left\|u_{j}\right\|^{2}\right) \rightarrow 0$ and together with $\int_{\Omega_{j}\left(0, r^{\prime}\right)}\left|v_{j}\right|^{2} \rightarrow 0$ it follows that $\left|v_{j}\right|_{2}^{2} \rightarrow 0$. Therefore, $\left\|v_{j}\right\| \rightarrow 0$, which contradicts $\left\|v_{j}\right\|=1$. So $v^{+} \neq 0$. 
By (27)-(28) and the fact that $|v|_{2}^{2}=\left|v^{+}\right|_{2}^{2}+\left|v^{-}\right|_{2}^{2}$, one has

$$
\begin{aligned}
\left\|v^{+}\right\|_{0}^{2}-\left\|v^{-}\right\|_{0}^{2}-\omega \int_{\mathbb{R}} v^{2} & \leq\left\|v^{+}\right\|^{2}-\left\|v^{-}\right\|_{0}^{2}-\omega|v|_{2}^{2} \\
& \leq-(\omega-\bar{\lambda})\left|v^{+}\right|_{2}^{2}-\left\|v^{-}\right\|_{0}^{2} \\
& <0 .
\end{aligned}
$$

Then there exists a finite interval $I \subset \mathbb{R}$ such that

$$
\left\|v^{+}\right\|_{0}^{2}-\left\|v^{-}\right\|_{0}^{2}-\omega \int_{I} v^{2}<0 .
$$

Note that

$$
\begin{aligned}
\frac{\Phi\left(u_{j}\right)}{\left\|u_{j}\right\|^{2}} \leq & \frac{1}{2}\left(\left\|v_{j}^{+}\right\|_{0}^{2}-\left\|v_{j}^{-}\right\|_{0}^{2}\right)-\int_{I} \frac{W\left(t, u_{j}\right)}{\left\|u_{j}\right\|^{2}} \\
= & \frac{1}{2}\left(\left\|v_{j}^{+}\right\|_{0}^{2}-\left\|v_{j}^{-}\right\|_{0}^{2}-\omega \int_{I}\left|v_{j}\right|^{2}\right) \\
& -\int_{I} \frac{W\left(t, u_{j}\right)-(\omega / 2)\left|u_{j}\right|^{2}}{\left\|u_{j}\right\|^{2}} \\
\leq & \frac{1}{2}\left(\left\|v_{j}^{+}\right\|_{0}^{2}-\left\|v_{j}^{-}\right\|_{0}^{2}-\omega \int_{I}\left|v_{j}\right|^{2}\right) \\
& +\frac{\omega r^{2}|I|}{2\left\|u_{j}\right\|^{2}},
\end{aligned}
$$

where $|I|$ denotes the Lebesgue measure of $I$. Combining this with (30) and (35), one has

$$
\begin{aligned}
0 & \leq \lim _{j \rightarrow \infty}\left(\frac{1}{2}\left\|v_{j}^{+}\right\|_{0}^{2}-\frac{1}{2}\left\|v_{j}^{-}\right\|_{0}^{2}-\int_{\Omega} \frac{W\left(t, u_{j}\right)}{\left\|u_{j}\right\|^{2}}\right) \\
& \leq \frac{1}{2}\left(\left\|v^{+}\right\|_{0}^{2}-\left\|v^{-}\right\|_{0}^{2}-\omega \int_{I} v^{2}\right)<0,
\end{aligned}
$$

which is a contradiction.

As a special case we have the following.

Lemma 9. Suppose that $\left(H_{1}\right)$ and $\left(H_{3}\right)-\left(H_{4}\right)$ are satisfied and $\rho>0$ is given by Lemma 7. Then, letting $e \in Y_{0}$ with $\|e\|=1$, there exists $r_{1}>0$ such that $\sup \Phi\left(\partial Q_{1}\right) \leq \kappa$, where $Q_{1}:=\{u=$ $\left.u^{-}+s e: u^{-} \in Q^{-}, s \geq 0,\|u\| \leq r_{1}\right\}$.

Lemma 10. Suppose that $\left(H_{1}\right)-\left(H_{4}\right)$ are satisfied. Then any $(C)_{c}$-sequence is bounded in $\mathrm{Q}$.

Proof. Let $\left\{u_{j}\right\} \subset Q$ be such that

$$
\Phi\left(u_{j}\right) \longrightarrow c, \quad\left(1+\left\|u_{j}\right\|\right) \Phi^{\prime}\left(u_{j}\right) \longrightarrow 0 .
$$

Then, for $C_{0}>0$,

$$
C_{0} \geq \Phi\left(u_{j}\right)-\frac{1}{2} \Phi^{\prime}\left(u_{j}\right) u_{j}=\int_{\mathbb{R}} \widetilde{W}\left(t, u_{j}\right) .
$$

Suppose to the contrary that $\left\|u_{j}\right\|$ is unbounded. Setting $v_{j}=$ $u_{j} /\left\|u_{j}\right\|$, then $\left\|v_{j}\right\|=1$ and $\left|v_{j}\right|_{\mu} \leq c\left\|v_{j}\right\|=c$ for all $\mu \epsilon$ $[2, \infty)$. Passing to subsequence, $v_{j} \rightarrow v$ in $Q, v_{j} \rightarrow v$ in $L_{\text {loc }}^{\mu}$ for $\mu \in[2, \infty)$, and $v_{j}(t) \rightarrow v(t)$ for a.e., $t \in \mathbb{R}$.

Note that

$$
\begin{aligned}
o(1) & =\Phi^{\prime}\left(u_{j}\right)\left(u_{j}^{+}-u_{j}^{-}\right)=\left\|u_{j}\right\|_{0}^{2}-\int_{\mathbb{R}} W_{u}\left(t, u_{j}\right)\left(u_{j}^{+}-u_{j}^{-}\right) \\
& =\left\|u_{j}\right\|_{0}^{2}-\left\|u_{j}\right\|^{2} \int_{\mathbb{R}} \frac{W_{u}\left(t, u_{j}\right)}{\left\|u_{j}\right\|}\left(v_{j}^{+}-v_{j}^{-}\right) \\
& =\left\|u_{j}\right\|^{2}\left(\left\|v_{j}\right\|_{0}^{2}-\int_{\mathbb{R}} \frac{W_{u}\left(t, u_{j}\right)}{\left\|u_{j}\right\|}\left(v_{j}^{+}-v_{j}^{-}\right)\right) \\
& =\left\|u_{j}\right\|^{2}\left(1-\left|v_{j}\right|_{2}^{2}-\int_{\mathbb{R}} \frac{W_{u}\left(t, u_{j}\right)}{\left\|u_{j}\right\|}\left(v_{j}^{+}-v_{j}^{-}\right)\right) .
\end{aligned}
$$

From (40), we obtain

$$
\int_{\mathbb{R}}\left(\left|v_{j}\right|^{2}+\frac{W_{u}\left(t, u_{j}\right)\left(v_{j}^{+}-v_{j}^{-}\right)}{\left\|u_{j}\right\|}\right) \rightarrow 1 .
$$

Set, for $x \geq 0$,

$$
h(x):=\inf \left\{\widetilde{W}(t, u): t \in \mathbb{R}, u \in \mathbb{R}^{2 N} \text { with }|u| \geq x\right\} .
$$

By $\left(\mathrm{H}_{3}\right)$ and $\left(\mathrm{H}_{4}\right), h(x)>0$ for all $x>0$ and $h(x) \rightarrow \infty$ as $x \rightarrow \infty$.

Let

$$
C_{l}^{m}=\inf \left\{\frac{\widetilde{W}(t, u)}{|u|^{2}}: t \in \mathbb{R} \text { with } l \leq|u(t)|<m\right\} .
$$

Since $W(t, u)$ depends periodically on $t$ and $\widetilde{W}(t, u)>0$ if $u \neq 0$, one has $C_{l}^{m}>0$ and

$$
\widetilde{W}\left(t, u_{j}\right) \geq C_{l}^{m}\left|u_{j}\right|^{2} \quad \forall t \in \Omega_{j}(l, m),
$$

where $\Omega_{j}(l, m)$ is defined in (32). It follows from (32) and (39) that

$$
\begin{aligned}
C_{0} \geq & \int_{\Omega_{j}(0, l)} \widetilde{W}\left(t, u_{j}\right)+\int_{\Omega_{j}(l, m)} \widetilde{W}\left(t, u_{j}\right) \\
& +\int_{\Omega_{j}(m, \infty)} \widetilde{W}\left(t, u_{j}\right) \\
\geq & \int_{\Omega_{j}(0, l)} \widetilde{W}\left(t, u_{j}\right)+C_{l}^{m} \int_{\Omega_{j}(l, m)}\left|u_{j}\right|^{2} \\
& +h(m)\left|\Omega_{j}(m, \infty)\right| .
\end{aligned}
$$

Using (45) we obtain

$$
\left|\Omega_{j}(m, \infty)\right| \leq \frac{C_{0}}{h(m)} \longrightarrow 0,
$$


as $m \rightarrow \infty$ uniformly in $j$, and for any fixed $0<l<m$,

$$
\int_{\Omega_{j}(l, m)}\left|v_{j}\right|^{2}=\frac{1}{\left\|u_{j}\right\|^{2}} \int_{\Omega_{j}(l, m)}\left|u_{j}\right|^{2} \leq \frac{C_{0}}{C_{l}^{m}\left\|u_{j}\right\|^{2}} \longrightarrow 0,
$$

as $j \rightarrow \infty$. It follows from (46) that, for any $s \in[2,+\infty)$,

$$
\begin{aligned}
\int_{\Omega_{j}(m, \infty)}\left|v_{j}\right|^{s} & \leq\left(\int_{\Omega_{j}(m, \infty)}\left|v_{j}\right|^{2 s}\right)^{1 / 2} \cdot\left|\Omega_{j}(m, \infty)\right|^{1 / 2} \\
& \leq c\left|\Omega_{j}(m, \infty)\right|^{1 / 2} \longrightarrow 0,
\end{aligned}
$$

as $m \rightarrow \infty$ uniformly in $j$.

Let $0<\epsilon<1 / 5$. By $\left(\mathrm{H}_{2}\right)$ there is $l_{\epsilon}>0$ such that

$$
\left|W_{u}(t, u)\right|<\frac{\epsilon}{c}|u|
$$

for all $|u| \leq l_{\epsilon}$. Consequently,

$$
\begin{aligned}
\int_{\Omega_{j}\left(0, l_{\epsilon}\right)} \frac{W_{u}\left(t, u_{j}\right)\left(v_{j}^{+}-v_{j}^{-}\right)\left|v_{j}\right|}{\left|u_{j}\right|} & \leq \int_{\Omega_{j}\left(0, l_{\epsilon}\right)} \frac{\epsilon}{c}\left|v_{j}^{+}-v_{j}^{-}\right|\left|v_{j}\right| \\
& \leq \frac{\epsilon}{c}\left|v_{j}\right|_{2}^{2}<\epsilon
\end{aligned}
$$

for all $j$.

Set $\sigma^{\prime}:=\tau / 2$. By $\left(\mathrm{H}_{4}\right),(48)$, and Hölder inequality, we can take $m_{\epsilon} \geq r$ large enough such that

$$
\begin{aligned}
& \int_{\Omega_{j}\left(m_{\epsilon}, \infty\right)} \frac{W_{u}\left(t, u_{j}\right)\left(v_{j}^{+}-v_{j}^{-}\right)\left|v_{j}\right|}{\left|u_{j}\right|} \\
& \leq\left(\int_{\Omega_{j}\left(m_{\epsilon}, \infty\right)} \frac{\left|W_{u}\left(t, u_{j}\right)\right|^{\sigma}}{\left|u_{j}\right|^{\sigma}}\right)^{1 / \sigma} \\
& \quad \times\left(\int_{\Omega_{j}\left(m_{\epsilon}, \infty\right)}\left(\left|v_{j}^{+}-v_{j}^{-}\right|\left|v_{j}\right|\right)^{\sigma^{\prime}}\right)^{1 / \sigma^{\prime}} \\
& \leq\left(\int_{\Omega_{j}\left(m_{\epsilon}, \infty\right)} c_{1} \widetilde{W}\left(t, u_{j}\right)\right)^{1 / \sigma}\left(\int_{\mathbb{R}^{N}}\left(\left|v_{j}^{+}-v_{j}^{-}\right|\right)^{\tau}\right)^{1 / \tau} \\
& \quad \times\left(\int_{\Omega_{j}\left(m_{\epsilon}, \infty\right)}\left|v_{j}\right|^{\tau}\right)^{1 / \tau} \\
& \leq \epsilon
\end{aligned}
$$

for all $j$. Note that there is $C=C(\epsilon)>0$ independent of $j$ such that $\left|W_{u}\left(t, u_{j}\right)\right| \leq C\left|u_{j}\right|$ for $t \in \Omega_{j}\left(l_{\epsilon}, m_{\epsilon}\right)$. By (47) there is $j_{0}$ such that

$$
\begin{aligned}
\int_{\Omega_{j}\left(l_{\epsilon}, m_{\epsilon}\right)} \frac{W_{u}\left(t, u_{j}\right)\left(v_{j}^{+}-v_{j}^{-}\right)\left|v_{j}\right|}{\left|u_{j}\right|} & \leq C \int_{\Omega_{j}\left(l_{\epsilon}, m_{\epsilon}\right)}\left|v_{j}^{+}-v_{j}^{-}\right|\left|v_{j}\right| \\
& \leq C\left|v_{j}\right|_{2}\left(\int_{\Omega_{j}\left(l_{\epsilon}, m_{\epsilon}\right)}\left|v_{j}\right|^{2}\right)^{1 / 2} \\
& \leq \epsilon
\end{aligned}
$$

for all $j \geq j_{0}$. By (50)-(52), one has

$$
\limsup _{j \rightarrow \infty} \int_{\mathbb{R}} \frac{W_{u}\left(t, u_{j}\right)\left(v_{j}^{+}-v_{j}^{-}\right)}{\left\|u_{j}\right\|} \leq 3 \epsilon .
$$

On the other hand, by (48), take $m_{\epsilon}>0$ large enough such that

$$
\int_{\Omega_{j}\left(m_{\epsilon}, \infty\right)}\left|v_{j}\right|^{2} \leq \epsilon
$$

Similar to (47), one has

$$
\int_{\Omega_{j}\left(0, m_{\epsilon}\right)}\left|v_{j}\right|^{2}=\frac{1}{\left\|u_{j}\right\|^{2}} \int_{\Omega_{j}\left(0, m_{\epsilon}\right)}\left|u_{j}\right|^{2} \leq \frac{m_{\epsilon}^{2}\left|\Omega_{j}\left(0, m_{\epsilon}\right)\right|}{\left\|u_{j}\right\|^{2}} \leq \epsilon .
$$

Thus (54) and (55) imply that

$$
\limsup _{j \rightarrow \infty} \int_{\mathbb{R}}\left|v_{j}\right|^{2} \leq 2 \epsilon
$$

By (53) and (56), one has

$$
\limsup _{j \rightarrow \infty} \int_{\mathbb{R}}\left(\left|v_{j}\right|^{2}+\frac{W_{u}\left(t, u_{j}\right)\left(v_{j}^{+}-v_{j}^{-}\right)}{\left\|u_{j}\right\|}\right) \leq 5 \epsilon<1,
$$

which contradicts (41). The proof is complete.

Let $\left\{u_{j}\right\} \subset Q$ be a $(C)_{c}$-sequence of $\Phi$; by Lemma 10, it is bounded, up to a subsequence; we may assume $u_{j} \rightarrow u$ in $Q$, $u_{j} \rightarrow u$ in $L_{\text {loc }}^{\mu}$ for $\mu \in[2, \infty)$, and $u_{j}(t) \rightarrow u(t)$ a.e. on $\mathbb{R}$. Plainly, $u$ is a critical point of $\Phi$. Set $u_{j}^{1}=u_{j}-u$.

Lemma 11. Under the assumptions of Theorem 2, one has, as $j \rightarrow \infty$,
(1) $\Phi\left(u_{j}^{1}\right) \rightarrow c-\Phi(u)$;
(2) $\Phi^{\prime}\left(u_{j}^{1}\right) \rightarrow 0$.

Proof. The proof of this lemma is similar to the one of Lemma 5.7 in [9] (or see [10]), so we omit it here.

Let $\mathscr{K}:=\left\{u \in Q: \Phi^{\prime}(u)=0, u \neq 0\right\}$ be the set of nontrivial critical points of $\Phi$.

Lemma 12. Under the assumptions of Theorem 2, the following two conclusions hold:

(1) $\phi:=\inf \{\|u\|: u \in \mathscr{K}\}>0$;

(2) $\psi:=\inf \{\Phi(u): u \in \mathscr{K}\}>0$.

Proof. (1) For any $u \in \mathscr{K}$, there holds

$$
0=\Phi^{\prime}(u) u^{+}=\left\|u^{+}\right\|_{0}^{2}-\int_{\mathbb{R}} W_{u}(t, u) u^{+},
$$

together with (25), which implies that

$$
\left\|u^{+}\right\|_{0}^{2} \leq \epsilon\left|u^{+}\right|_{2}^{2}+C_{\epsilon}\left|u^{+}\right|_{p}^{p} \leq c \epsilon\left\|u^{+}\right\|_{0}^{2}+c C_{\epsilon}\left\|u^{+}\right\|_{0}^{p},
$$


where $p \geq \tau$. Choose $\epsilon$ small enough; one has

$$
0<\left(\frac{1-c \epsilon}{c C_{\epsilon}}\right)^{2 /(p-2)} \leq\left\|u^{+}\right\|_{0}^{2}
$$

for each $u \in \mathscr{K}$. Therefore,

$$
\|u\|^{2}>\|u\|_{0}^{2}=\left\|u^{+}\right\|_{0}^{2}+\left\|u^{-}\right\|_{0}^{2}>\left(\frac{1-c \epsilon}{c C_{\epsilon}}\right)^{2 /(p-2)}>0 .
$$

(2) Suppose to the contrary that there exists a sequence $\left\{u_{j}\right\} \subset$ $\mathscr{K}$ such that $\Phi\left(u_{j}\right) \rightarrow 0$.

Then

$$
\begin{gathered}
0=\left\|u_{j}\right\|_{0}^{2}-\int_{\mathbb{R}} W_{u}\left(t, u_{j}\right)\left(u_{j}^{+}-u_{j}^{-}\right), \\
o(1)=\Phi\left(u_{j}\right)=\Phi\left(u_{j}\right)-\frac{1}{2} \Phi^{\prime}\left(u_{j}\right) u_{j}=\int_{\mathbb{R}} \widetilde{W}\left(t, u_{j}\right) .
\end{gathered}
$$

By (1), $\left\|u_{j}\right\| \geq \phi$. Clearly $\left\{u_{j}\right\}$ is a $(C)_{0}$-sequence of $\Phi$ and hence is bounded by Lemma 10. Using (63) and the discussion in the proof of Lemma 10, we see that, for any $0<l<m$ and $p \in[\tau, \infty), \int_{\Omega_{j}(l, m)}\left|u_{j}\right|^{2} \rightarrow 0$ and $\int_{\Omega_{j}(m, \infty)}\left|u_{j}\right|^{p} \rightarrow 0$ as $j \rightarrow \infty$. Therefore, it follows from (24) and (62) that for any $\epsilon^{\prime}>0$

$$
\underset{j \rightarrow \infty}{\lim \sup }\left\|u_{j}\right\|_{0}^{2} \leq \epsilon^{\prime}
$$

which contradicts (61). The proof is complete.

In the following lemma we discuss further the $(C)_{c}$ sequence. Let $[k]$ denote the integer part of $k \in \mathbb{R}$. The following lemma is standard by using Lemmas 11 and 12 (see $[1,19])$.

Lemma 13. Under the assumptions of Theorem 2, let $\left\{u_{j}\right\} \subset Q$ be a $(C)_{c}$-sequence of $\Phi$. Then either

(i) $u_{j} \rightarrow 0$ (and hence $c=0$ ), or

(ii) $c \geq \psi$ and there exist a positive integer $k \leq[c / \psi]$, $y_{1}, \ldots, y_{k} \in \mathscr{K}$, and sequence $\left\{a_{j}^{i}\right\} \subset \mathbb{Z}, i=1,2, \ldots, k$, such that, after extraction of a subsequence of $\left\{u_{j}\right\}$,

$$
\left\|u_{j}-\sum_{i=1}^{k} a_{j}^{i} * y_{i}\right\| \longrightarrow 0, \quad \sum_{i=1}^{k} \Phi\left(y_{i}\right)=c
$$

and for $i \neq q$,

$$
\left|a_{j}^{i}-a_{j}^{q}\right| \longrightarrow \infty
$$

as $j \rightarrow \infty$.

\section{Proof of Theorem 2}

In order to apply the abstract Theorems 5 and 6 to $\Phi$, we choose $X=Q^{-}$and $Y=E^{+} . X$ is separable and reflexive and let $\mathcal{S}$ be a countable dense subset of $X^{*}$. First we have the following.
Lemma 14. $\Phi$ satisfies $\left(\mathcal{N}_{0}\right)$.

Proof. We first show that $\Phi_{a}$ is $\mathscr{T}_{S}$-closed for every $a \in \mathbb{R}$. Consider a sequence $\left\{u_{j}\right\}$ in $\Phi_{a}$ which $\mathscr{T}_{S}$-converges to $u \in Q$ and write $u_{j}=u_{j}^{-}+u_{j}^{+}$and $u=u^{-}+u^{+}$. Observe that $\left\{u_{j}^{+}\right\}$ converges to $u^{+}$in norm topology. Since $W(t, u) \geq 0$, there exists $c>0$ such that

$$
\frac{1}{2}\left\|u_{j}^{-}\right\|_{0}^{2}=\frac{1}{2}\left\|u_{j}^{+}\right\|_{0}^{2}-\Phi\left(u_{j}\right)-\int_{\mathbb{R}} W\left(t, u_{j}\right)<c .
$$

Therefore, $\left\|u_{j}^{-}\right\|_{0}$ is bounded. So $\left\|u_{j}\right\|_{0}$ is bounded. Now we claim that $\left|u_{j}^{-}\right|_{2}$ is bounded. Assume by contradiction that $\left|u_{j}^{-}\right|_{2}$ is unbounded. Clearly, $\left\|u_{j}\right\|$ is also unbounded. Setting $v_{j}=u_{j} /\left\|u_{j}\right\|$, then $\left\|v_{j}\right\|=1$. Passing to subsequence, $v_{j} \rightarrow v$ in $Q, v_{j} \rightarrow v$ in $L_{\mathrm{loc}}^{\mu}$ for $\mu \in[2, \infty)$. Note that

$$
\int_{\mathbb{R}} W\left(t, u_{j}\right)<-a+\frac{1}{2}\left\|u_{j}^{+}\right\|_{0}^{2}-\frac{1}{2}\left\|u_{j}^{-}\right\|_{0}^{2}
$$

Therefore, $\int_{\mathbb{R}} W\left(t, u_{j}\right)$ is bounded and $\int_{\mathbb{R}}\left(W\left(t, u_{j}\right) /\left\|u_{j}\right\|^{2}\right) \rightarrow$ 0 . Similar to Lemma 8, we can obtain (33). From (33) and $\int_{\mathbb{R}}\left(W\left(t, u_{j}\right) /\left\|u_{j}\right\|^{2}\right) \rightarrow 0$ and together with $\int_{\Omega_{j}(0, r)}\left|v_{j}\right|^{2} \rightarrow 0$ it follows that $\left|v_{j}\right|_{2} \rightarrow 0$. Since $1=\left\|v_{j}\right\|^{2}=\left\|v_{j}\right\|_{0}^{2}+$ $\left|v_{j}\right|_{2}^{2}$, we obtain $\left\|v_{j}\right\|_{0} \rightarrow 1$, which contradicts the fact that $\left\|v_{j}\right\|_{0}=\left\|u_{j}\right\|_{0} /\left\|u_{j}\right\| \rightarrow 0$. So $\left|u_{j}^{-}\right|_{2}$ is bounded. Therefore, $\left\{u_{j}^{-}\right\}$is bounded in $Q$, and we have $u_{j}^{-} \rightarrow u^{-}$. Therefore, $u_{j} \rightarrow u$. It is easy to show that $\Psi$ is weakly sequentially lower semicontinuous. Thus, from the form of $\Phi$ it follows that $\Phi(u) \geq \lim \inf \Phi\left(u_{j}\right) \geq a$. So $u \in \Phi_{a}$ and $\Phi_{a}$ is $\mathscr{T}_{S^{-}}$ closed.

Next we show that $\Phi^{\prime}:\left(\Phi_{c}, \mathscr{T}_{\delta}\right) \rightarrow\left(Q^{*}, u^{*}\right)$ is continuous. It is sufficient to show that $\varphi^{\prime}$ has the same property. Let $u_{j} \rightarrow u$ in $Q$. Then $u_{j} \rightarrow u$ in $L_{\text {loc }}^{\mu}$ for $\mu \in$ $[2, \infty)$. It is obvious that

$$
\begin{array}{r}
\varphi^{\prime}\left(u_{j}\right) v=\int_{\mathbb{R}} W\left(t, u_{j}\right) v \longrightarrow \int_{\mathbb{R}} W(t, u) v=\varphi^{\prime}(u) v, \\
\forall v \in C_{0}^{\infty}(\mathbb{R})
\end{array}
$$

as $j \rightarrow \infty$. Now using the density of $C_{0}^{\infty}(\mathbb{R})$ in $Q$ one can obtain the desired conclusion.

Lemma 15. $\Phi$ satisfies $\left(\mathcal{N}_{1}\right)$.

Proof. We assume by contradiction that, for some $c_{1}>0$, there exists a sequence $\left\{u_{n}\right\}$ with $\Phi\left(u_{n}\right) \geq c_{1}$ and $\left\|u_{n}\right\|^{2} \geq$ $n\left\|u_{n}^{+}\right\|^{2}$. The form of $\Phi$ implies that

$$
\begin{aligned}
\left\|u_{n}^{-}\right\|^{2} & \geq(n-1)\left\|u_{n}^{+}\right\|^{2} \geq c(n-1)\left\|u_{n}^{+}\right\|_{0}^{2} \\
& \geq c(n-1)\left(2 c_{1}+\left\|u_{n}^{-}\right\|_{0}^{2}+2 \int_{\mathbb{R}} W\left(t, u_{n}\right)\right) .
\end{aligned}
$$

Since $c, c_{1}>0$ and $W\left(t, u_{n}\right) \geq 0$, it follows that $\left\|u_{n}^{-}\right\| \rightarrow \infty$ as $n \rightarrow \infty$. Hence $\left\|u_{n}\right\| \rightarrow \infty$. Set $v_{n}=u_{n} /\left\|u_{n}\right\|$. Then $\left\|v_{n}\right\|=1$ 
and $\left\|v_{n}^{+}\right\|^{2}=\left\|u_{n}^{+}\right\|^{2} /\left\|u_{n}\right\|^{2} \leq 1 / n \rightarrow 0$. Therefore, $v_{n} \rightarrow v=v^{-}$ in $Q$. By (70) we have

$$
1 \geq\left\|v_{n}^{-}\right\|^{2} \geq c(n-1)\left(\frac{2 c_{1}}{\left\|u_{n}\right\|^{2}}+\left\|v_{n}^{-}\right\|_{0}^{2}+2 \int_{\mathbb{R}} \frac{W\left(t, u_{n}\right)}{\left\|u_{n}\right\|^{2}}\right) .
$$

Thus $\left\|v_{n}^{-}\right\|_{0}^{2} \leq 1 / c(n-1) \rightarrow 0$. By (31)-(32) and (71), one has

$$
\begin{aligned}
\frac{1}{2 c(n-1)} & \geq \int_{\mathbb{R}} \frac{W\left(t, u_{n}\right)}{\left\|u_{n}\right\|^{2}} \geq \int_{\Omega_{n}(r, \infty)} \frac{W\left(t, u_{n}\right)}{\left\|u_{n}\right\|^{2}} \\
& \geq \omega \int_{\Omega_{n}\left(r^{\prime}, \infty\right)}\left|v_{n}\right|^{2},
\end{aligned}
$$

and together with $\int_{\Omega_{n}\left(0, r^{\prime}\right)}\left|v_{n}\right|^{2} \rightarrow 0$, it follows that $\left|v_{n}\right|_{2}^{2} \rightarrow$ 0 . Therefore, $\left\|v_{n}\right\| \rightarrow 0$, which is in contradiction with $\left\|v_{n}\right\|=$ 1. The proof is complete.

Now we give the proof of Theorem 2.

Proof. (1) Existence of a Homoclinic Orbit. With $X=Q^{-}$ and $Y=E^{+}$the condition $\left(\mathcal{N}_{0}\right)$ holds by Lemma 14 and $\left(\mathcal{N}_{1}\right)$ holds by Lemma 15 . Lemma 7 implies that $\left(\mathcal{N}_{2}\right)$ holds. Lemma 9 shows that $\Phi$ possesses the linking structure of Theorem 5. Therefore, there exists a sequence $\left\{u_{j}\right\} \subset Q$ such that

$$
\Phi\left(u_{j}\right) \longrightarrow c \geq \kappa, \quad\left(1+\left\|u_{j}\right\|\right) \Phi^{\prime}\left(u_{j}\right) \longrightarrow 0
$$

By Lemma $10\left\{u_{j}\right\}$ is bounded. Consequently, $\Phi^{\prime}\left(u_{j}\right) \rightarrow$ 0 . A standard argument shows that $\left\{u_{j}\right\}$ is a nonvanishing sequence; that is, there exist $\xi_{1}>0,0<R<\infty$, and $\left\{a_{j}\right\} \subset \mathbb{R}$ such that

$$
\liminf _{j \rightarrow \infty} \int_{a_{j}-R}^{a_{j}+R}\left|u_{j}\right|^{2} \geq \xi_{1}
$$

Setting $\tilde{u}_{j}(t)=u_{j}\left(t+a_{j}\right)$, by the invariance under translation of $\Phi,\left\{\widetilde{u}_{j}\right\}$ is a $(C)_{c}$-sequence of $\Phi$ and $\tilde{u}_{j} \rightarrow \widetilde{u}$. From

$$
\liminf _{j \rightarrow \infty} \int_{-R}^{R}\left|\tilde{u}_{j}\right|^{2} \geq \xi_{1}>0
$$

we see that $\tilde{u} \neq 0$, and hence $\tilde{u}$ is a nontrivial critical point of $\Phi$. Following the idea of [15], we prove that $\widetilde{u}$ is a homoclinic orbit of (HS). By (23) and Lemma $4, W_{u}(t, u) \in L^{\mu}$ for all $\mu \geq 2$. Hence, a standard regularity theory of solutions of ordinary differential equations shows that $u \in H_{\mathrm{loc}}^{1} \cap L^{\mu}$ for all $\mu \geq 2$. By (HS) and (23),

$$
|\dot{u}|^{2} \leq c\left(|u|^{2}+|u|^{2(p-1)}\right)
$$

where $p \geq \tau$. So for all $\varrho \in \mathbb{R}$, by Hölder inequality, one has

$$
\int_{\varrho-1 / 2}^{\varrho+1 / 2}|\dot{u}|^{2} \leq c\left\{\left(\int_{\varrho-1 / 2}^{\varrho+1 / 2}|u|^{2(p-1)}\right)^{1 /(p-1)}+\int_{\varrho-1 / 2}^{\varrho+1 / 2}|u|^{2(p-1)}\right\} .
$$

Integrating from $\varrho-1 / 2$ to $\varrho+1 / 2$ the following equality,

$$
u(\varrho)=u(t)+\int_{t}^{\varrho} \dot{u}(s) d s,
$$

yields, using Hölder inequality

$$
|u(\varrho)| \leq c\left(\int_{\varrho-1 / 2}^{\varrho+1 / 2}|u|^{2(p-1)}\right)^{1 /(p-1)}+\left(\int_{\varrho-1 / 2}^{\varrho+1 / 2}|\dot{u}|^{2}\right)^{1 / 2} .
$$

Since $u \in L^{2(p-1)},(77)$ and (79) show that

$$
|u(\varrho)| \longrightarrow 0 \quad \text { as }|\varrho| \longrightarrow \infty
$$

that is, $u$ is a homoclinic orbit of (HS). The proof is complete.

(2) Multiplicity. Following the idea of [10], the proof will be completed in an indirect way; namely, we show that if

$$
\frac{\mathscr{K}}{\mathbb{Z}} \text { is a finite set, }
$$

then $\Phi$ has an unbounded sequence of critical values, a contradiction. We do this by checking that if (81) is true then $\Phi$ satisfies all the assumptions of Theorem 6.

By the assumption $W(t, u)$ is even in $u$, hence so is $\Phi$. $\Phi(0)=0$ is deduced from $\left(\mathrm{H}_{1}\right)$. Lemma 8 shows that $\Phi$ satisfies $\left(\mathcal{N}_{3}\right)$. Let $\mathscr{T}$ be a set consisting of arbitrarily chosen representatives of the $\mathbb{Z}$-orbits of $\mathscr{K}$. Then $\mathscr{T}$ is a finite set by (77), and since $\Phi$ is odd we may assume that $\mathscr{T}=-\mathscr{T}$. If $z \in \mathscr{K}$, then $\Phi(z) \geq \psi$ by (2) of Lemma 12 . Hence there exists $\psi \leq \chi$ such that

$$
\psi \leq \min _{\mathscr{T}} \Phi=\min _{\mathscr{K}} \Phi \leq \max _{\mathscr{K}} \Phi \leq \max _{\mathscr{T}} \Phi \leq \chi .
$$

For $k \in \mathbb{N}$ and a finite set $\mathscr{B} \subset Q$ we define

$$
(\mathscr{B}, k):=\left\{\sum_{i=1}^{j} a_{i} * u_{j}: 1 \leq j \leq k, a_{i} \in \mathbb{Z}, u_{j} \in \mathscr{B}\right\} .
$$

As in Coti Zelati and Rabinowitz $[1,19]$,

$$
\inf \left\{\left\|u-u^{\prime}\right\|: u, u^{\prime} \in(\mathscr{B}, k)\right\}>0 \text {. }
$$

Now we check $\left(\mathcal{N}_{4}\right)$. Given a compact interval $I \subset(0, \infty)$ with $s:=\max I$ we set $k=[d / \psi]$ and $\mathscr{C}:=(\mathscr{T}, k)$. We have $P^{+}(\mathscr{T}, k)=\left(P^{+} \mathscr{T}, k\right)$. Thus from $(84)$

$$
\inf \left\{\left\|u_{1}^{+}-u_{2}^{+}\right\|: u_{1}, u_{2} \in \mathscr{C}, u_{1}^{+} \neq u_{2}\right\}>0 .
$$

In addition, $\mathscr{C}$ is a $(C)_{I}$-attractor by Lemma 13 and $\mathscr{C}$ is bounded because $\|u\| \leq k \max \{\|\bar{u}\|: \bar{u} \in \mathscr{T}\}$ for all $u \in \mathscr{C}$. Therefore, by Theorem 6 , $\Phi$ has an unbounded sequence of critical values which contradicts the assumption (81), and hence $\Phi$ has infinitely many geometrically distinct homoclinic orbits.

\section{Conflict of Interests}

The authors declare that there is no conflict of interests regarding the publication of this paper. 


\section{Acknowledgments}

F. Li was supported by the National Natural Science Foundation of China (Grant no. 11201211), Shandong Natural Science Foundation (Grant no. ZR2012AL04), and AMEP of Linyi University. J. Sun was supported by the National Natural Science Foundation of China (Grant no. 11201270 and no. 11271372), Shandong Natural Science Foundation (Grant no. ZR2012AQ010), and Young Teacher Support Program of Shandong University of Technology.

\section{References}

[1] V. Coti Zelati and P. H. Rabinowitz, "Homoclinic orbits for second order Hamiltonian systems possessing superquadratic potentials," Journal of the American Mathematical Society, vol. 4, no. 4, pp. 693-727, 1991.

[2] M. Izydorek and J. Janczewska, "Homoclinic solutions for a class of the second order Hamiltonian systems," Journal of Differential Equations, vol. 219, no. 2, pp. 375-389, 2005.

[3] M. Izydorek and J. Janczewska, "Homoclinic solutions for nonautonomous second order Hamiltonian systems with a coercive potential," Journal of Mathematical Analysis and Applications, vol. 335, no. 2, pp. 1119-1127, 2007.

[4] J. Sun, H. Chen, and J. J. Nieto, "Homoclinic solutions for a class of subquadratic second-order Hamiltonian systems," Journal of Mathematical Analysis and Applications, vol. 373, no. 1, pp. 2029, 2011.

[5] G. Arioli and A. Szulkin, "Homoclinic solutions of Hamiltonian systems with symmetry," Journal of Differential Equations, vol. 158, no. 2, pp. 291-313, 1999.

[6] T. Bartsch and A. Szulkin, "Hamiltonian systems: periodic and homoclinic solutions by variational methods," in Handbook of Differential Equations: Ordinary Differential Equations, chapter 2, pp. 77-146, North-Holland, 2006.

[7] C.-N. Chen and X. Hu, "Maslov index for homoclinic orbits of Hamiltonian systems," Annales de l'Institut Henri Poincaré. Analyse Non Linéaire, vol. 240, no. 4, pp. 589-603, 2007.

[8] V. Coti Zelati, I. Ekeland, and E. Séré, "A variational approach to homoclinic orbits in Hamiltonian systems," Mathematische Annalen, vol. 288, no. 1, pp. 133-160, 1990.

[9] Y. Ding, Variational Methods for Strongly Indefinite Problems, vol. 7 of Interdisciplinary Applied Mathematics Science, World Scientific, 2007.

[10] Y. Ding, "Multiple homoclinics in a Hamiltonian system with asymptotically or super linear terms," Communications in Contemporary Mathematics, vol. 8, no. 4, pp. 453-480, 2006.

[11] Y. Ding and M. Girardi, "Infinitely many homoclinic orbits of a Hamiltonian system with symmetry," Nonlinear Analysis: Theory, Methods \& Applications, vol. 38, no. 3, pp. 391-415, 1999.

[12] Y. Ding and L. Jeanjean, "Homoclinic orbits for a nonperiodic Hamiltonian system," Journal of Differential Equations, vol. 237, no. 2, pp. 473-490, 2007.

[13] Y. Ding and C. Lee, "Existence and exponential decay of homoclinics in a nonperiodic superquadratic Hamiltonian system," Journal of Differential Equations, vol. 246, no. 7, pp. 2829-2848, 2009.

[14] Y. H. Ding and S. J. Li, "Homoclinic orbits for first order Hamiltonian systems," Journal of Mathematical Analysis and Applications, vol. 189, no. 2, pp. 585-601, 1995.
[15] Y. Ding and M. Willem, "Homoclinic orbits of a Hamiltonian system," Zeitschrift für Angewandte Mathematik und Physik, vol. 50, no. 5, pp. 759-778, 1999.

[16] J. Sun, J. Chu, and Z. Feng, "Homoclinic orbits for first order periodic Hamiltonian systems with spectrum point zero," Discrete and Continuous Dynamical Systems, vol. 33, no. 8, pp. 3807-3824, 2013.

[17] M. Willem and W. Zou, "On a Schrödinger equation with periodic potential and spectrum point zero," Indiana University Mathematics Journal, vol. 52, no. 1, pp. 109-132, 2003.

[18] T. Bartsch and Y. Ding, "Deformation theorems on nonmetrizable vector spaces and applications to critical point theory," Mathematische Nachrichten, vol. 279, no. 12, pp. 12671288, 2006.

[19] V. Coti Zelati and P. H. Rabinowitz, "Homoclinic type solutions for a semilinear elliptic PDE on $\mathbf{R}^{N}$," Communications on Pure and Applied Mathematics, vol. 45, no. 10, pp. 1217-1269, 1992. 


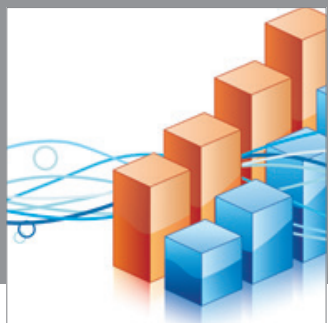

Advances in

Operations Research

mansans

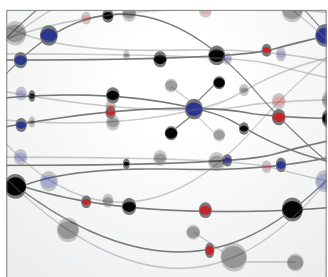

The Scientific World Journal
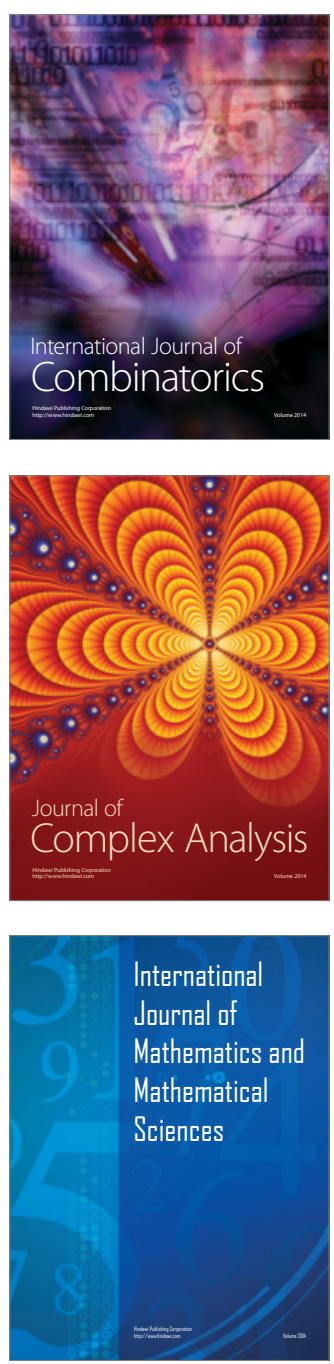
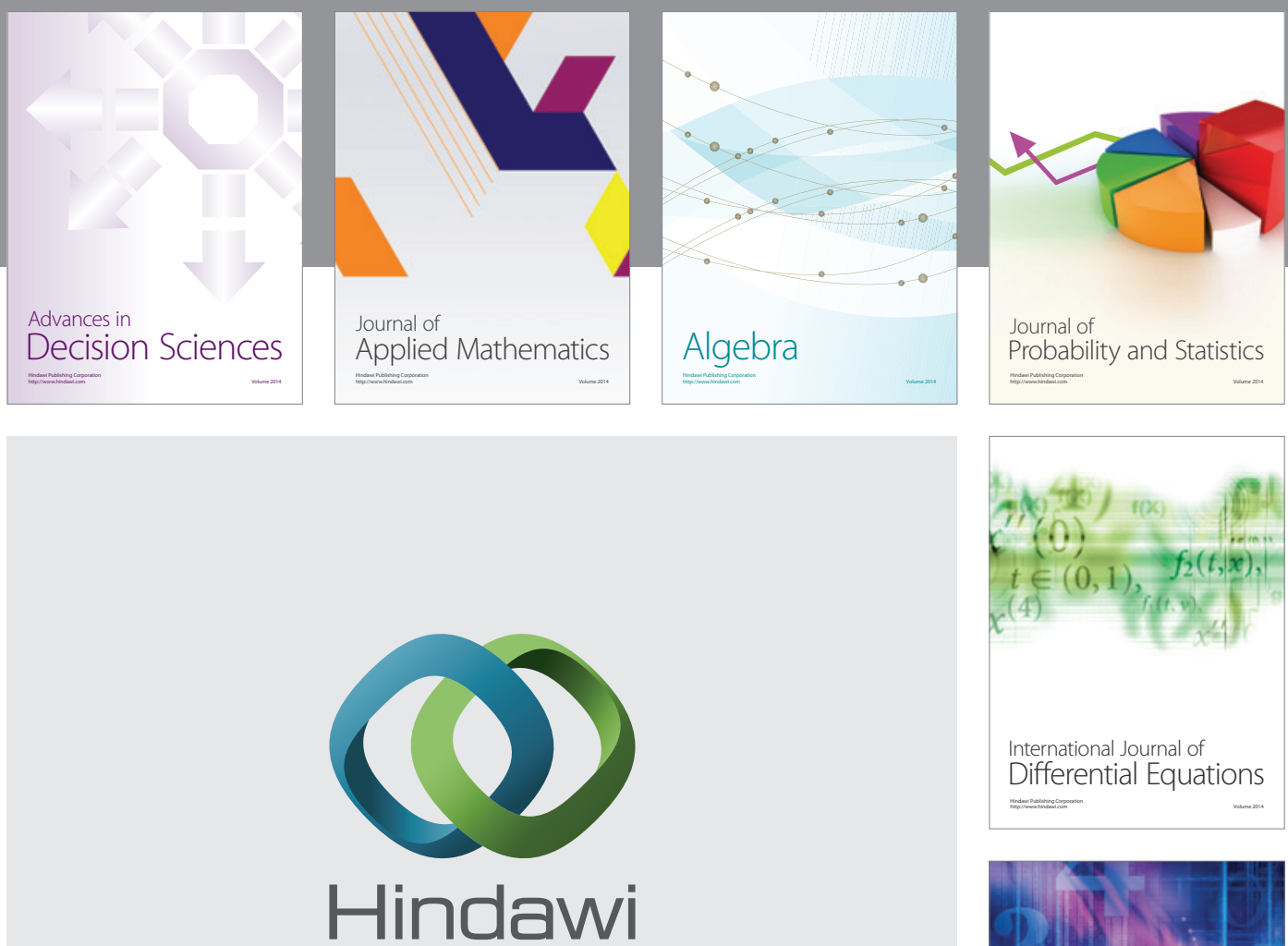

Submit your manuscripts at http://www.hindawi.com
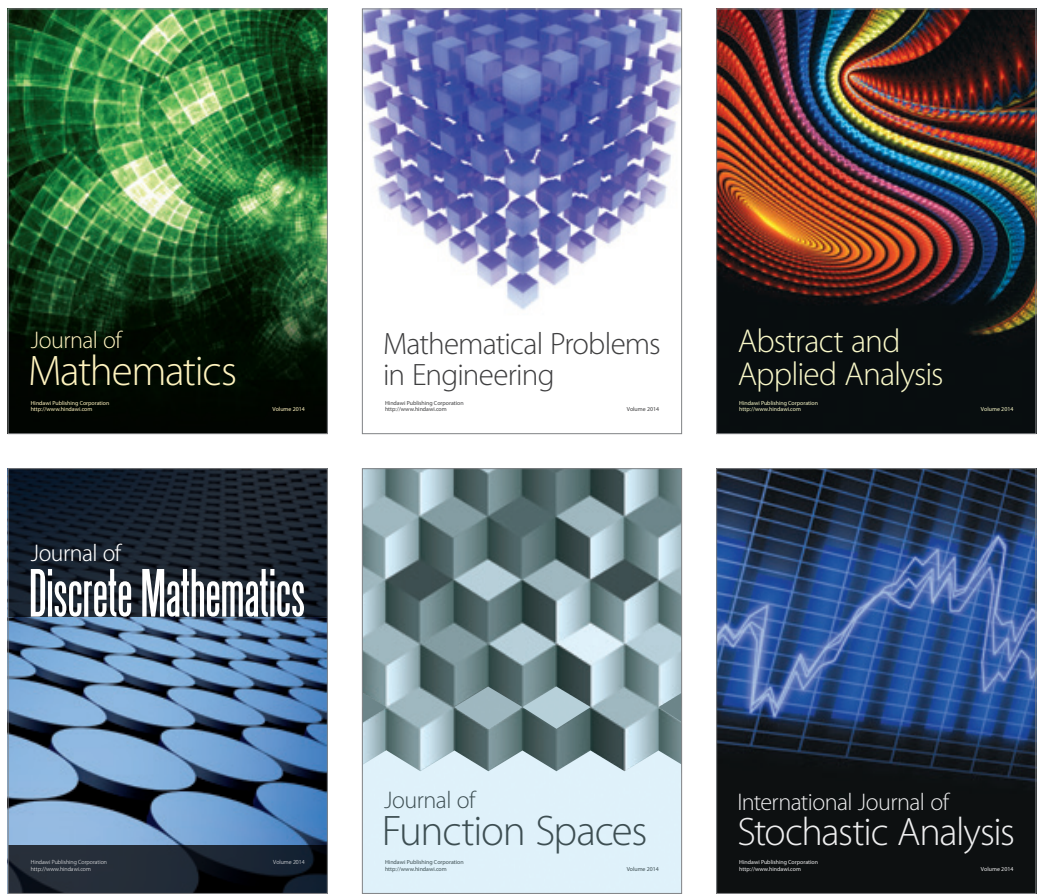

Journal of

Function Spaces

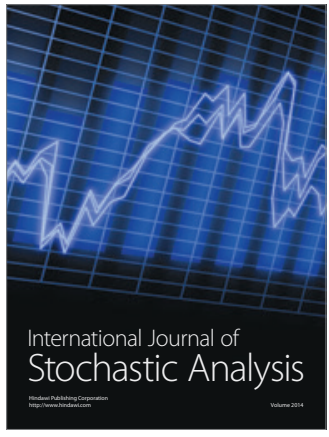

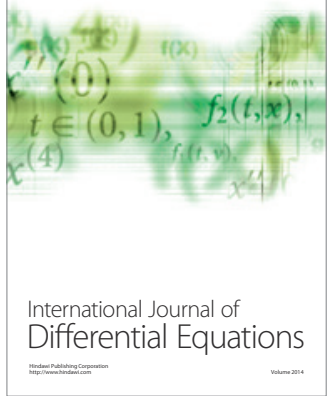
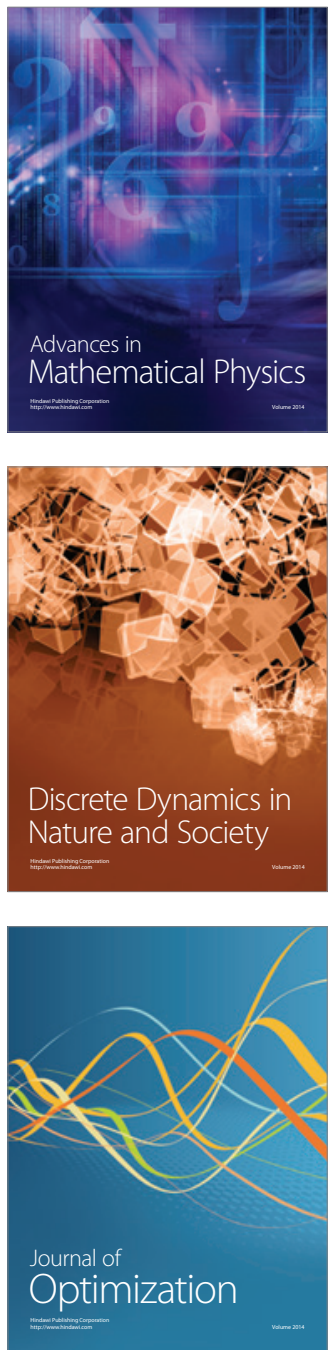